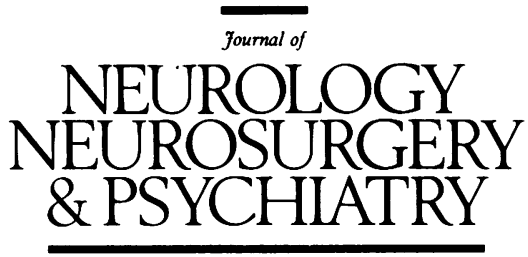

\title{
Editorial
}

\section{The treatment of primary malignant brain tumours}

The management of primary brain tumours is controversial with widely differing clinical attitudes, therapeutic approaches and results. A consensus report from a multinational cooperative group should provide a welcome and useful overview of current treatment approaches and ongoing and future avenues of research activity. The consensus report from the Brain Group of the European Organisation for Research and Treatment of Cancer (EORTC) goes some way in recounting new experimental treatment strategies but fails to address the important and controversial area of optimal clinical management of adult supratentorial gliomas. In contrast to medulloblastoma and primary cerebral lymphoma, where a multi-modality therapeutic approach is widely accepted, opinions about treatment of low and high grade gliomas differ widely ${ }^{1}$ and clinically acceptable guidelines would have great benefit.

The EORTC report divides primary brain tumours into high grade gliomas (glioblastoma and anaplastic astrocytoma), low grade astrocytomas, primary brain lymphomas and medulloblastomas. This is a clinically useful division as the results of treatment are different and patients with these neoplasms present to clinicians with varying experience and commitment to neuro-oncology.

Low grade astrocytomas are more common than the EORTC statement indicates. ${ }^{23}$ With the CT scan, the diagnosis of low grade astrocytoma or oligodendroglioma can be made with a high degree of confidence. Management decisions, however, and the need for histological confirmation, are subject to varying opinions by clinicians. The EORTC statement does not provide recommendations about choice of management and the role of surgery. CT imaged guided stereotaxic biopsy will provide tissue diagnosis with a mortality of $<1 \% .{ }^{4}$ Resection will be possible for some well circumscribed lesions in "silent areas" of the brain $^{56}$ and may alleviate troublesome epilepsy. ${ }^{7}$

Retrospective studies suggest that "total" or macroscopic excision of low grade glioma increases the prospect of long term survival. ${ }^{38}$ Many of these lesions, however, cannot be completely excised since they infiltrate extensively into normal brain. Several non-randomised studies suggest that postoperative radiotherapy increases the percentage of five and 10 year survivors. ${ }^{9-11}$ Prospective studies of early and delayed radiotherapy in low grade glioma are being conducted by the MRC and EORTC. Both groups are experiencing serious recruitment difficulties that reflect clinical and patient related prejudices. If these studies confirm that radiotherapy prolongs median disease free survival then further studies may be necessary to define optimal radiation schedules.

Many patients with a low grade glioma, regardless of treatment, survive for many years so that it is important to avoid morbidity as a result of treatment. The incidence of late onset psychomotor slowing due to treatment related leucoencephalopathy can be reduced by strict adherence to the limits of cerebral radiation tolerance and a reduction in the volume of irradiated brain. ${ }^{11}$ Stereotaxic external beam therapy could be helpful in selected circumstances, ${ }^{12}$ and shares with interstitial brachytherapy ${ }^{13}$ the advantage of a limited field of irradiation and rapid dose falloff. This invasive technique, however, requires surgery to remove radionecrotic tumour and brain in a large proportion of patients. ${ }^{14}$ Each of these interventions can cause focal neurological deficits.

The use of chemotherapy in low grade astrocytoma is controversial. These tumours have a low labelling index when tested using either BrdU or Ki-67..$^{15} 16$ The selection timing, and scheduling of cytotoxic agents is largely empirical. Assessment of response is difficult and chemotherapy has been shown to potentiate radiation leucoencephalopathy. The preservation of the blood-brain barrier in low grade gliomas ${ }^{17}$ impairs delivery of many cytotoxic agents. ${ }^{18}$

Histological diagnosis of primary brain lymphoma is now safely and readily obtained using CT imaged guided stereotaxic biopsy. ${ }^{4}$ There are uncertainties about the best treatment approach to this uncommon disorder. Patient age, tumour histology and multiplicity of lesions all influence survival. ${ }^{19}$ In the United Kingdom a randomised study evaluating radiotherapy and combination chemotherapy is being conducted by the MRC. This analytical assessment is essential if outcome is to be improved.

The EORTC statement on medulloblastoma summarises conventional approaches to management, results, current problems and uncertainties. Several large, multicentre, international trials have explored the question of chemotherapy in addition to optimal surgery and irradiation. ${ }^{20}$ The role of adjuvant and "sandwich" chemotherapy for low risk patients has not been proved. ${ }^{21}$ In children with high risk tumours (less than two years of age, incomplete surgical excision of tumour and with brain stem involvement) some gain in early survival is seen using chemotherapy. ${ }^{21}$ In very young patients chemotherapy may allow delayed irradiation and reduce related toxicity. ${ }^{22}$ Whether an increase in side effects as a result of the treatment modality will reduce benefit remains unknown.

The treatment of patients with glioblastoma or anaplastic astrocytoma remains one of the most challenging problems in clinical neuro-oncology. The EORTC statement overlooks many practical problems associated with management of these patients and concentrates on unorthodox treatment options presently under evaluation. The impact of treatment for high grade glioma becomes 
less and survival worse with increasing patient age, neurological disability and malignancy of the tumour. ${ }^{123-27}$ Since many patients with high grade glioma have a dramatic clinical response to glucocorticoids some clinicians accept a CT diagnosis of malignant glioma and recommend no further intervention. Steroid therapy can cause major morbidity with proximal myopathy, weight gain, acne, psychosis, dyspepsia and diabetes mellitus. ${ }^{28}$ In the elderly or severely disabled patient, such an approach to treatment may be practical but in five to $10 \%$ of cases CT diagnosis will be wrong. ${ }^{49}$

The role of surgery in high grade glioma needs to be defined. Stereotaxic CT imaged guided biopsy is generally a safe diagnostic tool ${ }^{40}$ but complication rates as high as $12 \%$ have been reported. ${ }^{26}$ Cytoreductive surgery relieves symptoms and signs of intracranial hypertension and improves postoperative functional status. ${ }^{2631}$ Extensive surgical resection may improve survival times ${ }^{27}$ but this effect is modest and the postoperative complication rate and 30 day case fatality may be as high as $20 \%{ }^{23}{ }^{26}$ Tumour debulking surgery results in substantial reduction in steroid requirements and a smoother clinical course during radiotherapy. Whether similar outcome can be achieved after stereotaxic biopsy followed by radiotherapy is unknown. ${ }^{32}$

The consensus document describes conventionally used dose and fractionation radiotherapy schedules for malignant glioma. Such therapy is necessary if median survival times of 12 months are to be obtained. ${ }^{25}$ Differing dosage and fractionation schedules are being evaluated, including shorter courses of treatment for the elderly or poor prognostic groups, ${ }^{33}$ but any further improvement in survival time is likely to be marginal. ${ }^{12}$ Interstitial brachytherapy can be used for the treatment of recurrences. Initial results are promising but this technique requires special resources and has complications that limit its general use. ${ }^{14}$

Single agent nitrosourea chemotherapy given orally or intravenously is as effective as multi-agent chemotherapy in high grade glioma. ${ }^{25}$ Whether this should be given as adjuvant or withheld until relapse is uncertain, although short term benefits with adjuvant therapy can be seen. ${ }^{34}$ No guidance or preference about timing of chemotherapy is given by the EORTC, and the optimal number of adjuvant treatment courses is unknown. Toxicity with nitrosoureas is mainly due to myelosuppression, and is cumulative. ${ }^{25} \mathrm{At}$ present a convincing increase on survival is not obtained either by high dose chemotherapy followed by autologous bone marrow rescue or by intracarotid administration of cytotoxic drugs. Both treatments are hazardous. ${ }^{2035}$ Although tumour levels of the cytotoxic agent can be increased by intracarotid delivery, the drug delivery to normal brain rises disproportionately and increases the risk of leucoencephalopathy.

The role of immunotherapy in patients with a malignant glioma who relapse after conventional treatment is now the subject of intense study. ${ }^{36-38}$ Initial studies using interferon, interleukins, lymphokine activated killer cells (LAK) and divalent antibodies have been complicated by a high incidence of side effects and show mixed, but generally disappointing clinical responses. ${ }^{36} 39$

All currently available therapies are palliative. The goal of treatment is to provide the longest period of good quality life. This may be jeopardised by tumour or treatment related neuropsychological and functional deficits. ${ }^{3}$ Decisions about patient management are best made at a multidisciplinary neuro-oncology clinic. The combination of expertise enables evaluation of new treatments and inclusion of patients in clinical trials. ${ }^{3}$ Regular audit may disclose areas of treatment inefficiency. ${ }^{23}$ The surgical neuro-oncologist is likely to use stereotaxic techniques, to have low surgical morbidity and to be capable of innovative approaches to glioma surgery. ${ }^{464041}$ The radiation and medical neuro-oncologist will be aware of the limitations and potential toxicity of both conventional and unconventional treatments. ${ }^{25}$ Assessment of the achievement of a neuro-oncology clinic only by the percentage of 18 month or two year survivors alone may be inappropriate and prospective assessments should address the question of quality of outcome. Improving both the treatment of the tumour and the patient's quality of life provides the challenge of neuro-oncology.

IAN R WHITTLE ANNA GREGOR

Department of Clinical Neurosciences,

Western General Hospital,

Edinburgh

1 Wroe SJ, Foy PM, Shaw MDM, et al. Neurological and neurosurgical approaches in management of malignant brain tumours. $B M$ 1986;293:1015-18.

2 Russell D, Rubenstein LJ. Pathology of tumours of the central nervous system. 5th ed. London: Edward Arnold, 1989:1012.

3 Mahaley MS, Mettlin C, Natarajan N, et al. National survey of patterns of care for brain tumour patients. J Neurosurg 1989;71:826-36.

4 Thomas DGT, Nouby RM. Experience in 300 cases of CT-directed stereotaxic surgery for lesion biopsy and aspiration of hematoma. Brit $J$ Neurosurg 1989;3:337-42.

5 Yasargil MG, Teddy PJ, Roth P. Selective amygdalohippocampectomy. Operative anatomy, and surgical technique. Adv Tech Stand Neurosurg 1985;12:93-122.

6 Sabin HI, Whittle IR. Stereotaxic craniotomy and intraoperative lesion localisation; Technical note. Acta Neurochir (Wien) 1990;102:137-40.

7 Goldring S, Rich KM, Picker S. Experience with gliomas in patients presenting with achronic seizure disorder. Clin Neurosurg 1985;33:15-42.

8 Laws E, Taylor WF, Clifton MB, et al. Neurosurgical management of low grade astrocytomas of the cerebral hemisphere. J Neurosurg 1984;61 665-73.

9 Leibel SA, Sheline G. Radiation therapy for neoplasms of the brain. J Neurosurg 1987;66:1-22.

10 Lindegaard K-F, Mork SJ, Eide GE, et al. Statistical analysis of clinicopathological features, radiotherapy and survival in 170 cases of oligodendroglioma. J Neurosurg 1987;67:224-30.

11 Morantz RA. Radiation therapy in the treatment of cerebral astrocytoma Neurosurg 1987;20:975-82.

12 Brada $\mathrm{M}$. Back to the future-radiotherapy in high grade gliomas. $\mathrm{Br} J$ Cancer 1989;60:1-4.

13 Gutin PH, Phillips TL, Wara WM, et al. Brachytherapy of recurrent malignant brain tumours with removable high activity iodine-125 sources. $J$ Neurosurg 1984;60:61-70.

14 Gutin PH, Leibel SA, Wara WM, et al. Recurrent malignant glioma; Survival following interstitial brachytherapy with high activity iodine-125 sources. J Neurosurg 1987;67:864-73.

15 Hoshino T, Rodriguez LA, Cho KG, et al. Prognostic implications of the proliferative potential of low grade astrocytomas. $J$ Neurosur 1988;69:839-42.

16 Raghaven R, Steart PV, Weller RO. Cell proliferation patterns in the diagnosis of astrocytomas, anaplastic astrocytomas and glioblastoma multiforme: a Ki-67 study. Neuropathol Applied Neurobiol 1990;16: multiforme

17 Seitz RJ, Wechsler W. Immunohistochemical demonstration of serum proteins in human cerebral gliomas. Acta Neuropathol (Berl) 1987;73: 145-52.

18 Workman P. The pharmacology of brain tumour chemotherapy. In: Bleehan NM, ed. Tumours of the brain. Berlin: Springer-Verlag, 1986:182-200.

19 Hochberg EH, Miller DC. Primary central nervous system lymphoma. $J$ Neurosurg 1988;68:835-55.

20 Kornblith PL, Walker M. Chemotherapy for malignant glioma. J Neurosurg 1988;68:1-17.

21 Bloom HJG. Treatment of brain gliomas in children. In: Bleehan NM, ed. Tumours of the brain. Berlin: Springer-Verlag, 1986:121-40.

22 Packer RJ, Sutton LN, Atkins TE, et al. A prospective study of cognitive function in children receiving whole brain radiotherapy and chemofunction in children receiving whole brain radiother
therapy. Two year results. $J$ Neurosurg 1989;70:707-13.

23 Denholm S, El Shunnar K, Whittle IR. What is optimal management for patients over 60 years with supratentorial glioma? Lessons from an audit. patients over 60 years with supratentorial gl
$J$ Neurol Neurosurg Psychiatry 1990;53:444.

24 Shapiro WR. Therapy of adult malignant brain tumours. What have the clinical trials taught us. Semin Oncol 1986;13:38-45.

25 Shapiro WR, Green SB, Burger PC, et al. Randomized trial of three chemotherapy regimens and two radiotherapy regimens in postoperative treatment of malignant glioma. $J$ Neurosurg 1989;71:1-9.

26 Vecht Ch J, Avezaat CJ, van Putten WLJ, et al. The influence of the extent of surgery on the neurological function and survival in malignant glioma. A retrospective analysis in 243 patients. J Neurol Neurosurg Psychiatry 1990;53:466-71.

27 A report of the MRC Brain Tumour Working party. Prognostic factors for high guide malignant gliomas. Development of a prognostic index. J Neuro-oncol 1990;8:243-7.

28 Weissman DE, Dufer D, Vogel V, et al. Corticosteroid toxicity in neurooncology patients. J Neuro-oncol 1987;5:125-8.

29 Choksey MS, Valentine A, Shawdon H. Computerised tomography in the diagnosis of malignant brain tumours. Do all require biopsy? J Neurol Neurosurg Psychiatry 1989;52:821-5.

30 Apuzzo ML, Sabshin JK. Computed tomographic guidance stereotaxis in 
the management of intracranial mass lesions. Neurosurgery 1983;12: 277-85.

31 Cohadon F. Indications for surgery in the management of gliomas. Adv Tech Stand Neurosurg 1990;17:189-234.

32 Coffey RJ, Dunsford LD, Taylor FH. Survival after stereotaxic biopsy of malignant gliomas. Neurosurgery 1988;22:465-73.

33 Newall J, Ransohoff J, Kaplan B. Glioblastoma in the older patient: how long a course of radiotherapy is necessary; J Neuro-oncol 1988;6:325-7.

34 Stenning SP, Friedman LS, Bleehan NM. An overview of published results from randomized studies of nitrosoureas in primary high grade mignant from randomized studies of nitrosourea

35 Loew F, Papavero $L$. The intra-arterial route of drug delivery in the chemotherapy of malignant brain tumours. Adv Tech Stand Neurosurg 1988;16(11A):51-79.
36 Sawamura Y, de Tribolet N. Immunobiology of brain tumours. Adv Tech Stand Neurosurg 1990;19:4-44.

37 Merchant RE, Ellison MD, Young HF. Immunotherapy for malignant glioma using recombinant interleukin-2 and activated autologous lymphocytes. $J$ Neuro-oncol 1990;8:173-88.

38 Nitta $\mathrm{T}$, Sato $\mathrm{K}$, Okumura $\mathrm{K}$, et al. Induction of cytotoxicity in human Tcells coated with anti-glioma $\mathrm{x}$ anti-CD3 bispecific antibody against cells coated with anti-glioma $\mathrm{x}$ anti-CD3 bispecif

39 Nitta T, Sato K, Yagita H, et al. Preliminary trial of specific targeting therapy against malignant glioma. Lancet 1990;335:368-70.

40 Kaye AH, Morstyn G, Apuzzo ML. Photoradiation therapy and its potential in the management of neural tumours. J Neurosurg 1988;69:1-14.

41 Kelly PJ, Kall BA, Goerss S, et al. Computer assisted stereotaxic laser resection of intraaxial brain neoplasms. J Neurosurg 1986;69:423-39.

\section{Neurological stamp}

\section{Mandragora}

Mandragora (Mandrake) was used as a pain killer in ancient times. It is a herb of the nightshade (Solanacea). The scent of the mandrake fruit was reputed to alleviate headaches and insomnia. Hippocrates noted that a small dose would relieve anxiety and the deepest depression. If drunk its hypnotic properties allowed amputation or cauterisation. It was also used as an aphrodisiac and cure for sterility.

The roots bore a resemblance to the human figure. Gathering the mandrake was not without danger as the plant when uprooted uttered a shriek which caused death or insanity in those who heard it. One way to collect the herb was to dig around the roots leaving only a small portion. A hungry dog was then tied to it. Food placed beyond the length of the rope encouraged the dog to strain at the leash and uproot the mandrake. The dog died following the shriek of the plant which was now harmless.

A stamp commemorating the Seventh European Congress of Anaesthesiology in 1986 reproduced a medieval illustration from the Austrian National Library. This shows the mandrake plant with its human like roots about to be uprooted. A worried man is departing hurriedly before the fatal shriek is emitted. (Stanley Gibbons 2103, Scott 1360).

L F HAAS 